\title{
Antifascisti tra carcere, clandestinità ed esilio. La "riscoperta" dei carteggi
}

\section{Sara Galli}

Storicamente, 1 (2005).

ISSN: 1825-411X. Art. no. 18. DOI: 10.12977/stor523

«Annali Istituto Gramsci Emilia - Romagna», 1, 1997, Bologna, Clueb, 1998, pp. 282;

Massimo Mila, Argomenti strettamente familiari. Lettere dal carcere 19351940, a cura di Paolo Soddu, Introduzione di Claudio Pavone, Torino, Einaudi, 1999, pp. LXIII, 792;

Angelo Tasca, A Cécile, a cura di Sergio Anelli e Sergio Soave, Torino, Aragno, 2001, pp.124;

Dalla seconda metà degli anni Ottanta, è stata rilevata in modo assai incisivo, da parte di taluni studiosi, l'incapacità dei paradigmi storiografici affermatisi a partire dal secondo dopoguerra di restituire la complessità che ha contrassegnato le alterne e talora contraddittorie vicende legate all'antifascismo. Si è avvertita quindi l'esigenza di avviare un dibattito che, prendendo le mosse dalle lacune della storiografia esistente, ha sollecitato una riflessione più ampia intorno ai fattori che maggiormente hanno influito sugli esiti della ricerca storica, delimitandone in parte i percorsi[[1]].

Nel contesto politico, nazionale ed internazionale che ha contraddistinto i decenni successivi al secondo dopoguerra, sebbene in modo nient'affatto lineare, la storiografia legata ai partiti di tradizione antifascista è parsa investita dal compito di legittimare quella tavola di valori che avevano animato, seppur in modo diverso, l'antifascismo e la Resistenza, e che della Repubblica costituivano il necessario fondamento[[2]]. Ne è risultato, in 
generale, il predominio di una storia politica per lo più incentrata sulle idee, le strategie e le azioni promosse dai partiti e dalle organizzazioni antifasciste, tanto in Italia quanto nel variegato mondo dell'emigrazione politica. Una letteratura, questa, che si è in parte distinta per lo sforzo di ripercorrere in modo sempre più preciso e puntuale la storia dell'antifascismo e che, pur andando via via arricchendosi di ricerche dalla solida base documentaria e dallo spiccato rigore metodologico, ha a lungo continuato a risentire, in misura differente, di quell'originario "mandato".

A distanza di oltre quarant'anni, alcuni storici hanno dunque ritenuto necessario mettere in discussione sia l'attualità di quei vecchi paradigmi sia i presupposti che ne avevano disegnato il profilo. La caduta del muro di Berlino e il successivo smembramento dell'Unione Sovietica, stravolgendo un universo simbolico ed un sistema di significati la cui sedimentazione aveva profondamente influenzato la storiografia, non potevano che accelerare questo processo di revisione in chiave problematica di categorie e modelli esistenti, allargando in molteplici direzioni gli orizzonti della ricerca storica.

Negli anni Novanta si sono distinte, soprattutto per la novità delle proposte che li qualificavano, alcune ricerche rivolte ad una dimensione più propriamente sociale, nonché allo stretto intreccio tra sfera pubblica e privata che caratterizzava i percorsi degli antifascisti. In tal modo si è espressa l'esigenza di approfondire, attraverso l'utilizzo di fonti di carattere eterogeneo, taluni aspetti della biografia di uomini e donne o di interi nuclei familiari che alla lotta al fascismo si erano dedicati con passione e talvolta con vera abnegazione. Le scritture private, le memorie inedite, i frammenti di autonarrazione, ma soprattutto le corrispondenze di tipo personale, oltre che politico, fonti, queste, a lungo ignorate dalla precedente storiografia, hanno rivelato tutta la loro portata al fine di aprire nuovi territori di indagine. Questi studi hanno evidenziato la complessità, nonché la drammaticità della scelta compiuta dagli antifascisti, mettendo validamente in discussione l'immagine monolitica che la memorialistica ne aveva spesso restituito. A questa 
tendenza si è poi accompagnata, in alcuni casi, un'attenzione ed una sensibilità particolari alle differenze di genere, che nel contesto antifascista definivano in modo piuttosto netto anche gli ambiti dell'azione politica[[3]].

Il bisogno di oltrepassare il confine che tradizionalmente separava la dimensione pubblica da quella privata, sanzionando il primato dell'una sull'altra, per sondare anche l'universo dei sentimenti e delle passioni che avevano animato i protagonisti di quelle complesse vicende è altresì testimoniato dall'attenzione che parte della storiografia ha dimostrato per $\mathrm{i}$ carteggi di diversi esponenti dell'antifascismo, la cui edizione, specie negli ultimi anni, ha conosciuto un certo slancio[[4]]. Gli epistolari, infatti, permettendo di verificare il codice linguistico, i metodi comunicativi, i procedimenti letterari utilizzati, così come la "naturale" accettazione, l'elaborazione o la messa in discussione, da parte di chi scriveva, di consolidati ruoli di genere, forniscono allo studioso una chiave di lettura privilegiata[[5]]. Benché si avvalga di regole e modelli predeterminati, contemplando altresì la «coesistenza di più tempi»[[6]], la scrittura epistolare possiede inoltre quell'immediatezza che consente di seguire un percorso nel suo divenire, prima che la riflessione a posteriori - come avviene nelle memorie successive - abbia operato una ricostruzione del passato più spesso funzionale alle esigenze e alle scelte di un presente che volge il suo sguardo al futuro. 
Per questi motivi le corrispondenze, specie allorquando rappresentavano il solo mezzo di comunicazione tra persone legate da sentimenti profondi, consentono di cogliere le esitazioni, le espressioni di turbamento, così come le incertezze o le contraddizioni di quegli antifascisti che si apprestavano, di volta in volta, a raccontare e a raccontarsi alle persone più care. Attraverso queste fonti lo studioso, costretto a confrontarsi con questioni assai spinose che, investendo la dimensione più umana ed esistenziale, richiedono di essere valutate con grande cautela, può tentare di ripercorrere taluni percorsi biografici di antifascisti, o di concentrarsi su esperienze particolari, tentando di restituirne appieno la complessità [[7]].

Nel 1998 usciva un numero degli «Annali dell'Istituto Gramsci Emilia Romagna», interamente dedicato al Carteggio Paolo Betti - Lea Giaccaglia, conservato presso il medesimo Istituto[[8]]. Tale fondo è in buona parte composto dalla corrispondenza intercorsa tra la coppia di comunisti bolognesi Paolo e Lea negli anni compresi tra il 1922 e il 1935[[9]]. Sposati e con due figli, questi militanti avevano dovuto conoscere, come spesso accadeva ai comunisti attivi in Italia, il dolore di prolungate separazioni, dovute alla reclusione, ora dell'uno, ora di entrambi, nelle carceri fasciste. Risale dunque a quei lunghi anni la maggior parte della corrispondenza dei due coniugi, che affidavano alle lettere i loro pensieri, nonché il desiderio di preservare la propria vita affettiva.

«L'unico fascino del nostro amore è ora tutto su questo misero foglietto scriveva Lea a Paolo nel 1928 -, ed il cuore se ne pasce ed è insaziabilmente bisognoso di libare a tale fonte. I giorni passano terribilmente lunghi e sembra che essi alimentino la febbre che ci divora di stringere al nostro petto gli adorati pupi. Sento, sento il bisogno delle loro carezze di udire la loro adorata vocetta invocarmi, di perdere il mio tempo a farli belli e a trastullarli, a insegnare loro tante cose [...]»[[10]].

Se la condanna a numerosi anni da scontare in prigione veniva accettata dai comunisti come una possibile variabile della loro esistenza, così come la 
divisione dai compagni di vita o dalla famiglia d'origine, più dura doveva risultare la rinuncia a vedere crescere i figli e a star loro vicini. La vicenda di Paolo Betti e Lea Giaccaglia si rivela in tal senso particolarmente drammatica, in quanto nel 1928, mentre entrambi erano detenuti nelle carceri fasciste, Luce, la loro primogenita, inviata l'anno prima dalla madre, allora funzionaria di Partito, a vivere in Unione sovietica, si ammalò gravemente e morì. Quell'evento, sul quale si tornerà pure in seguito, doveva gettare Lea, che ne fu messa al corrente solo nel 1930, nella più cupa disperazione, minandola tanto nella salute fisica quanto in quella psichica[[11]].

A questo proposito, Mariuccia Salvati, nell'introduzione al volume, evidenzia come nel contesto della clandestinità il peso della militanza doveva riversarsi sull'universo maschile e quello femminile in modo assai diverso. Aspetto, questo, che dalla tragica vicenda di Paolo e Lea, affiora in modo significativo.

«[...] l'antifascismo fu, per le militanti comuniste una scelta che condizionò la loro esistenza, condannandole a una solitudine ben più aspra di quella maschile, perché non trovava sintonia e consolazione neppure nel proprio mondo di appartenenza; la scelta investiva infatti la cultura femminile per eccellenza, quella dei «legami famigliari » e ne sanciva la rottura anziché, come è naturale nella vita femminile, la cura e la conservazione» (19).

Attraverso il Carteggio Betti Giaccaglia è possibile quindi assistere al dipanarsi di una complessa trama politica, personale e familiare, nella quale la corrispondenza assume, di volta in volta, valori e significati del tutto particolari. Alle modalità con cui questa coppia tentava di imprimere un andamento continuo alla propria vita affettiva, tramite il rapporto epistolare, sono dedicati, pur nella loro diversità, i saggi che accompagnano le lettere pubblicate, poste in coda al volume[[12]].

Patrizia Gabrielli, nel suo intervento dal titolo «Lunga sarà la nostra attesa» (23-61), si concentra sulla dimensione più privata e quotidiana dei due comunisti, tentando di fare luce, non solo sugli effetti che la repressione 
aveva prodotto sulle loro esistenze, ma anche sul progressivo modificarsi del loro rapporto, nella misura in cui la lontananza e la reclusione doveva separarli in modo sempre più netto.

Gli scenari che si profilano sono di volta in volta diversi: ad un primo periodo compreso tra il 1923 e il 1927, nel quale Lea rimaneva in libertà, dividendosi tra le responsabilità familiari (tra cui la nascita di un secondo figlio) e l'attività politica, mentre Paolo subiva taluni periodi di allontanamento e di reclusione, seguivano, dopo l'ingresso della Giaccaglia nella clandestinità, gli anni in cui entrambi si sarebbero trovati prima in carcere e poi al confino.

Attraverso l'esame del carteggio, Gabrielli riesce ad individuare con grande acutezza i momenti nei quali Paolo e Lea, ognuno saldamente ancorato ad un preciso modello di genere, riuscivano a rassicurarsi a vicenda, ma nondimeno le fasi in cui, in seguito a particolari contingenze, si operavano dei significativi ribaltamenti di ruoli, causa di altrettanti inquietudini ed attriti. Ciò era accaduto quando Lea, sempre più impegnata nell'attività illegale del Partito, si era trovata a dover diradare la corrispondenza diretta al coniuge, così come la metodica assistenza che gli aveva prestato fino ad allora, discostandosi in tal modo dall'immagine assai confortante di sposa e madre premurosa che nel corso degli anni aveva conservato.

Le fratture createsi nel rapporto con Paolo, dopo l'entrata nella dimensione clandestina, avevano potuto ricomporsi solo in seguito all'arresto e alla reclusione di Lea, che a quel punto veniva a trovarsi in una situazione per alcuni versi simmetrica rispetto al marito[[13]]. Entrambi in prigione, i coniugi Betti, uniti dal sacrificio che la fede politica aveva imposto loro, potevano comunicare sulla base di un'esperienza simile ed affidarsi alla parola scritta, al fine di continuare a svolgere il ruolo di genitori. Carla Tonini, nel suo saggio Educare sorvegliare e amare. L'educazione all'infanzia nelle lettere ai figli di Paolo Betti e Lea Giaccaglia (63-89), analizza proprio il rapporto che, tramite la corrispondenza, Lea e Paolo riuscirono a mantenere con i figli, soffermandosi sulle linee generali di quello che si presenta come una sorta 
di comune progetto educativo[[14]].

La figura di Lea, nel lungo periodo, pare emergere dal carteggio come l'asse portante della famiglia. Insegnante di professione, Giaccaglia, a differenza del marito, aveva conciliato in modo continuativo la cura e la crescita dei bambini al proprio lavoro, per poi riprendere, a partire circa dal 1925, l'attività politica. Nei diversi momenti in cui Paolo, prima per mancanza di lavoro, poi per motivi politici, si era trovato lontano, Lea non aveva mancato di informarlo circa i problemi inerenti all'educazione dei figli, soffermandosi spesso su talune questioni generali di pedagogia, connesse anche al suo lavoro. Al tempo stesso, parlava costantemente ai bambini del padre, nell'intento di mantenere vivo un sentimento e un ricordo che il tempo rischiava di indebolire. II modo in cui Giaccaglia interpretava il proprio ruolo materno, osserva Tonini, lungi dall'appiattirsi su modelli esclusivamente tradizionali, si arricchiva quindi di significati assai vasti, mutuati spesso anche dalla propria fede politica. 
II 1927 aveva segnato per la famiglia Betti uno spartiacque definitivo, sancendo la separazione di Lea, divenuta a tutti gli effetti un funzionario di Partito, tanto da Luce, mandata con alcuni compagni in Unione Sovietica, quanto da Vero, il secondogenito, affidato alle cure della nonna materna. L'arresto e l'incarcerazione, avvenuti in quello stesso autunno, dovevano poi impedire per molti anni a Giaccaglia di ricongiungersi col figlio, il quale avrebbe potuto raggiungerla a Ponza, dove si trovava al confino, solo nel 1934; mentre di Luce, morta poco dopo, doveva rimanerle solo il ricordo dolorosissimo. Logorata dai sensi di colpa per quella grave perdita, della quale fu messa al corrente solo nei due anni a seguire, Lea - rileva Tonini nella corrispondenza diretta al marito non doveva comunque cessare di occuparsi dell'educazione morale e scolastica da impartire a Vero. Dalle lettere al figlio, evidenzia Tonini, emerge, in particolare, la determinazione di Paolo ad esercitare una funzione di guida e al tempo stesso di controllo sia sulle letture sia sui comportamenti del bambino, incitato a più riprese ad osservare una precisa disciplina.

«Quindi caro mio Vero - scriveva Paolo al figlio nel 1932 - come hai già cominciato a fare, raccontami sempre tutto. Dimmi pure proseguendo su questa buona via cosa pensi di te, di me, di tutti, di ogni cosa liberamente, io poi ti scriverò quanto è buono bello e quanto invece è da combattersi; cercherò di essere chiaro perché tu comprenda e ne sia convinto, ma se per la tua tenera età non vi riuscirai ancora ricordati che il tuo babbino, per la gioia, supremo diritto specialmente di tutti i bimbi, t'indica la via che devi seguire e che ti porterà preparato per la vera vita».[[15]] 
Maggiormente libero il rapporto con la madre, con la quale Vero, una volta giunto a Ponza, doveva trascorrere due anni in un clima di relativa serenità e spensieratezza, in compagnia dei figli degli altri confinati, e «a diretto contatto con l'ambiente politico e morale che i genitori ritenevano più adatto» (88). Purtroppo anche quel periodo doveva terminare drammaticamente, quando Lea, nel 1936, da tempo debilitata, moriva a causa di una grave infezione.

Il carteggio Betti Giaccaglia, consentendo di cogliere lo strettissimo nesso tra dimensione politica e personale di una coppia di comunisti, rivela tutta la sua ricchezza anche nel fornire elementi preziosi ai fini di un'indagine sulla formazione, nonché sulla cultura politica di una generazione di militanti che dal Partito socialista erano passati, sin dall'anno della sua fondazione, al Pcd'I[[16]].

Simona Urso, attraverso le pagine del suo saggio Scriversi, leggersi, leggere (1923-1934). Pratiche comunicative e carcere nel Fondo Betti-Giaccaglia (91-164), propone un'analisi attenta ed assai innovativa delle letture affrontate da Paolo e Lea nel corso di oltre un decennio. I riferimenti ai libri letti, posseduti o desiderati, di cui la corrispondenza tra i due coniugi è molto ricca, risalgono ai periodi di detenzione prima di Paolo poi di entrambi. Come è noto, per i comunisti, la reclusione rappresentava infatti un'opportunità per migliorare la propria preparazione teorica, in vista di un prossimo ritorno all'attività di Partito.

Urso, nel prendere in considerazione le letture dei coniugi Betti, così come la loro evoluzione nel corso del tempo, tenta, in particolar modo, di «rilevare somiglianze, discrepanze e sovrapposizioni fra l'eredità politica della militanza socialista e la nuova cultura politica che si viene formando con l'adesione al Pcd'l» (96). Attenta alle differenze di genere, la studiosa non manca inoltre di soffermarsi sulle diverse modalità che sembravano connotare il rapporto di Lea con la lettura rispetto al marito.

Attraverso il carteggio di Paolo e Lea, Urso tenta innanzitutto di tracciare un 
inventario della biblioteca di casa Betti, nonché di quella carceraria dei due coniugi. I libri posseduti dai due comunisti, così come il loro percorso culturale, paiono indicare la grande rilevanza che l'esperienza all'interno della Federazione giovanile socialista bolognese ebbe sulla loro formazione. In particolare, sottolinea Urso, sembra possibile mettere a fuoco "una cultura costruitasi attraverso l'incontro tra suggestioni sindacalrivoluzionarie [...] che si innesta[va]no sul mito della rivoluzione russa, affiancate però ad un apparato teorico acquisito alla scuola di positivismo, del marxismo secondinternazionalista dell'Editrice Avanti! e della U.[niversità] P.[opolare]» (120).

I riferimenti contenuti nelle lettere dal carcere hanno nondimeno consentito alla studiosa di procedere ad una prima valutazione dei libri letti da Paolo e Lea, a partire dalle case editrici. Come si ricava dal saggio, lungi dal presentare il profilo del lettore medio, i coniugi Betti, formatisi soprattutto sui classici prodotti dall'attività editoriale socialista, sembrano avere seguito la traiettoria culturale di quella generazione che, dopo aver subito il fascino del positivismo, era stata partecipe della crisi dei suoi paradigmi.

Rintracciando il percorso letterario di Paolo e Lea, lungo tutto il periodo coperto dal carteggio, Urso evidenzia il profondo cambiamento di significato che, verso la fine degli anni Venti, quando entrambi si trovavano in prigione, la pratica della lettura era andata via via assumendo per i comunisti. Nonostante la presenza assai vincolante della censura carceraria, si era infatti imposta, secondo le direttive del Partito, una sorta di rigida ortodossia, che prevedeva per i militanti un'attività di lettura strettamente finalizzata alla propria formazione politica.

A marcare in modo netto la differenza tra il retaggio socialista e la proposta, fortemente innovativa, originata dalla cultura terzinternazionalista erano, come evidenzia acutamente Ursola studiosa, i diversi modelli pedagogici elaborati dalle due parti: di tipo "gerarchizzante», nel primo caso, basato sul "controllo reciproco» e sulla "verifica reciproca del testo» nel secondo (152). 
Né Paolo né Lea paiono essere sfuggiti a questa disciplina, interpretata, a quanto pare, più liberamente da Giaccaglia. Ciò che comunque emerge con forza, nonostante le innegabili differenze che distinguono i percorsi di Paolo e Lea, è l'uniformità di un'esperienza politica, da entrambi vissuta in modo sentito e totalizzante, che ha reso la loro detenzione un ulteriore momento di condivisione del proprio percorso.

Se nella propria corrispondenza tanto Paolo Betti quanto Lea Giaccaglia esprimevano l'estrema necessità del loro sacrificio, ben diverso e più problematico si rivela il rapporto epistolare tra gli antifascisti rinchiusi in carcere e quei familiari che non condividevano le loro scelte politiche. Assai importante, al fine di osservare il dispiegarsi ed il modificarsi della relazione tra un giovane antifascista detenuto e la madre, appare l'edizione, uscita nel 1999, della corrispondenza carceraria di Massimo Mila, divenuto nel secondo dopoguerra un musicologo di grande fama. Mila, giovane antifascista torinese, dopo essere stato ammonito nel 1929, a causa di una lettera scritta con alcuni compagni a Benedetto Croce, contenente valutazioni negative circa l'operato di Mussolini, nel 1935 era stato arrestato con l'accusa di fare parte di Giustizia e Libertà. Condannato dal Tribunale Speciale a sette anni di carcere, egli doveva dare avvio, dal momento della sua reclusione, ad un intenso scambio epistolare con la madre, Clelia Carena Mila, destinato ad interrompersi solo nel 1940, allorquando finiva di scontare la sua pena. A dare corpo al volume dal significativo titolo Argomenti strettamente familiari - i soli che la censura permetteva venissero trattati nella corrispondenza carceraria - le 318 lettere che Mila in quasi cinque anni di prigione aveva indirizzato alla madre.

Claudio Pavone, nella sua introduzione, individua con grande sensibilità i nodi centrali della corrispondenza tra Mila e Clelia Carena, soffermandosi sugli aspetti più controversi che sembravano connotare il rapporto tra $\mathrm{i}$ detenuti antifascisti ed i propri cari. In particolare, doveva spesso rivelarsi doloroso il confronto tra chi, come Mila, tentava di vivere la propria reclusione con dignità, considerandola un possibile esito delle proprie scelte 
politiche, e coloro che, al pari di sua madre, ritenevano invece che la rinuncia alla libertà in nome dei propri ideali fosse un sacrificio decisamente eccessivo, o addirittura inutile.

Emblematica del travaglio interiore che accompagnava molto spesso i detenuti politici è la vicenda, ripercorsa da Pavone, relativa alla richiesta di grazia che Mila, oggetto delle continue pressioni familiari, aveva inoltrato nel 1935 a Mussolini. Le numerose contraddizioni contenute in quel documento, la cui scorrettezza procedurale - osserva Pavone - doveva invalidarlo a priori, consentono di assistere, al pari di diverse lettere risalenti a quel periodo, al sorgere di incertezze, titubanze ed inquietudini che andavano talvolta a scontrarsi con il desiderio di adottare una condotta coerente. Doveva essere la condanna, accompagnata dall'instaurarsi con altri compagni di Giustizia e Libertà, tra cui Renzo Giua e Vittorio Foa[17], di sentimenti di reciproca comprensione e fraterna amicizia, a placare, almeno in parte, le ansie del giovane Mila e ad avere su di lui quello che Pavone definisce un "effetto catartico» (XVIII). Nel 1937 infatti Mila scriveva alla madre quanto per lui fosse preferibile scontare la propria pena in uno stato di relativa serenità, piuttosto che avventurarsi in gesti suscettibili di provocare ulteriori tormenti interiori.

"forse nessuno che non sia stato in galera riuscirà a capire questo, e tu non lo crederai o lo troverai ingiusto e crudele da parte mia, ma io ti confesso che preferisco di molto conservare quella calma e tranquillità d'animo che faticosamente mi procuro, anche all'ipotesi di vedere ridotta o magari cessata la mia pena, ma a costo di nuove turbolenze, di nuovi inquietudini, nuovi affanni, di nuove incertezze»[[18]]

Costante sembra invece rimanere, per il giovane antifascista, al pari di molti altri detenuti politici, la difficoltà di comunicare l'esperienza carceraria a chi non l'aveva sperimentata, nonché la conseguente frustrazione per il mancato o inadeguato sostegno dei propri cari nei momenti più delicati. $A$ ricorrere quasi ossessivamente nelle lettere di Mila è il bisogno di impartire 
alla madre, nonché alla fidanzata Francesca, indiretta destinataria di alcune missive, indicazioni molto precise su come soddisfare le proprie esigenze, quasi ad esprimere l'estrema necessità di mantenere, almeno in parte, una sorta di controllo sul mondo esterno. Altrettanto frequenti si susseguivano le sfuriate dovute a quelli che Mila definiva gli "eccessi di zelo» della madre, che, nel tentativo di prestare lui aiuto materiale, finiva per oltrepassare abbondantemente le sue richieste.

"Il pensiero di voi non è per me un conforto, ma un tormento, perché Dio solo sa quali cretinerie siate capaci di pensare e di combinare nella vostra esagerazione esaltata.

Per evitare altre fesserie, ti avverto che qualunque altra cosa io ricevessi, non richiesta espressamente da me, la consegnerò immediatamente al cappellano che faccia delle distribuzioni di biancheria ai detenuti bisognosi. [...]. E se continuerò a ricevere lettere troppo idiote e deprimenti, smetterò per pace mia, di leggerle; e smetterò di scrivervi, perché non vale la pena se devo solo farmi cattivo sangue»[[19]]

Accadeva infatti spesso, come sottolinea anche Pavone, che i familiari riversassero incautamente su chi già stava affrontando la dura prova del carcere la propria angoscia, suscitando nei detenuti sensi di colpa e sofferenze supplementari. A questo proposito, Mila, non senza una buone dose di ironia, nel febbraio del 1936 domandava alla madre di mantenere un contegno nonché un atteggiamento coraggiosi, al fine di potere entrambi superare nel modo migliore quella pena reciproca.

"Scusatemi se sono brontolone, ma non ho tanti motivi di stare allegro, e purtroppo è difficile che me ne portino le vostre lettere. Non ci pensi mai dove andremmo a finire se facessi anch'io come fate voi, e mi mettessi a coltivare e a descrivervi per filo e per segno, in bella forma letteraria, tutti gli abbattimenti, le nostalgie, le impazienze e le disperazioni che mi tocca superare? Non rimarrebbe altro, per voi e per me, che attaccarci tutti quanti un cordino al collo, e attaccare il cordino alquanto in alto, coll'aiuto di una 
sedia, da respingere poi con un calcio. Allegria, allegria!»[[20]]

Ad influire sui contenuti e sulla forma delle lettere inviate dal carcere contribuivano senza dubbio la censura, che il detenuto, quando scriveva, aveva sempre presente, e l'autocensura, che si manifestava appunto in una forma di pudore o di reticenza a far mostra delle proprie debolezze o dei propri dispiaceri. A questo proposito, Giuliano Pajetta, rifacendosi anche alla propria esperienza scriveva:

"Fin dal primo giorno eri tu il tuo censore. Quello che c'era di noia nel carcere, quello che c'era di tristezza in un giorno o in un mese quando ci si scontrava con una vita che ti isolava dal mondo [...] quello non si poteva, non si doveva scriverlo. Non potevi concedere al censore di spiare la tua debolezza o la tua amarezza, non potevi buttare debolezza e dolore sul tavolo di casa tua, farli pesare sul dolore e la debolezza di una madre, di una donna, di ragazzi che dovevano vivere la loro vita, una vita diversa da quella del carcere. Le nostre lettere, ognuno di noi lo sapeva, potevano esser chieste da un compagno, mostrate a un altro antifascista; e tu non dovevi commuovere sulle tue miserie, non dovevi spaventare nessuno a proposito di una vita che altri avrebbero dovuto avere il coraggio, persino la passione, di affrontare»[[21]]

Per Mila, evidenzia Paolo Soddu nella sua nota, si aggiungeva poi il cruccio di non riuscire a trovare nella famiglia il retroterra culturale e politico in cui collocare le origini del proprio percorso. Benché Clelia Carena avesse compiuto, nella propria vita, scelte coraggiose ed anticonformiste, le sue inclinazioni politiche non erano state affatto estranee a quella parte della piccola borghesia che aveva assistito all'imporsi del fascismo con un certo compiacimento.

Nella sue lettere Mila pare determinato ad accorciare le distanze ideali dalla madre, da sempre considerate un grosso ostacolo, attraverso la proposta di un continuo confronto su temi ed argomenti dall'immediato riscontro politico. Nel tentativo, forse non sempre cosciente, di coinvolgere Clelia Carena nel 
proprio percorso culturale, il giovane finiva per investirla di una serie di oneri, tra cui quello di ricopiare o conservare i propri appunti e le proprie note in merito agli studi compiuti.

Tramite la lettura delle missive prende via via corpo la figura di una madre che, nel tenace desiderio di avvicinarsi al figlio e di sostenerlo, non esitava a spedire lui, oltre i libri richiesti, saggi, poesie ed articoli da lei selezionati e ricopiati a mano. Clelia Carena doveva inoltre impegnarsi in un costante dialogo circa le opere lette da entrambi, ponendo di frequente al figlio domande e chiarimenti sui temi ed i concetti da lui esplicitati. Lo studio e le discussioni avviate con gli altri compagni di carcere dovevano dunque, non solo conferire al giovane antifascista una più solida preparazione politica, consentendo lui di compiere un percorso di riflessioni comune a molti militanti di Giustizia e libertà, ma anche divenire uno degli oggetti principali della corrispondenza con la madre.

\section{A questo proposito Paolo Soddu osserva:}

"Attraverso le lettere noi scorgiamo un giovane uomo [...] ricercare attraverso le letture non solo le ragioni delle minoranze che avevano informato le fasi decisive della storia nazionale, ma anche quei molteplici fili che avevano legato l'Italia alla civiltà europea, dalla quale essa era andata nuovamente isolandosi con la dittatura fascista» (LX).

In particolare, come evidenzia Pavone, gli anni trascorsi in carcere furono per Mila animati da un continuo interrogarsi circa le basi dello storicismo idealista, e circa il concetto di libertà formulato da Croce, entrambi ritenuti insoddisfacenti ai fini di un'azione politica pratica. Nodi, questi, sui quali un'intera generazione di antifascisti si doveva confrontare, prospettando, nel dopoguerra, soluzioni e progettualità politiche differenti.

Senza dubbio, gli anni trascorsi nel chiuso delle carceri fasciste, dove si palesava il vero volto della repressione, avevano notevolmente inciso sulla personalità di Mila, che ritornava in libertà dopo un'esperienza personale, 
politica e morale davvero intensa. Sarebbe stata la Resistenza - rileva Pavone - a mettere alla prova, in circostanze del tutto nuove, la preparazione teorica e pratica accumulata dal giovane Mila, che doveva dare un rilevante contributo alla lotta di liberazione, all'interno di Giustizia e libertà.

Se la reclusione fu uno dei vari mezzi utilizzati dal fascismo, al fine di punire qualunque forma di dissenso, la violenza e le persecuzioni politiche, minacciando la vita ed il futuro di chi ne era fatto oggetto, dovevano indurre gli antifascisti a scelte tanto dolorose quanto inevitabili. Per molti l'esilio rappresentò l'unica ed estrema possibilità di sottrarsi ad un'esistenza divenuta ogni giorno più densa di incognite e di pericoli. Ricercati dalla Polizia, costretti a vivere ed agire nella clandestinità, furono molti coloro che si trovarono dolorosamente a dover rinunciare al proprio mondo d'appartenenza, per rifugiarsi in paesi dalle più solide tradizioni democratiche.

Il contesto dell'emigrazione antifascista, ancora per molti versi da indagare, si presenta come una sorta di mosaico composto da una grande varietà di percorsi politici, personali e familiari[[22]]. A mostrare taluni tratti distintivi assai peculiari, all'interno di tale ambito, è il fenomeno dell'emigrazione di funzionari e dirigenti comunisti. II loro, infatti, fu un esilio sottoposto a regole e dinamiche piuttosto rigide, definite, da un lato, dalla disciplina di Partito, e dall'altro dalle norme ed i sacrifici che imponevano i periodi più o meno lunghi di clandestinità. Per alcuni, l'emigrazione comportò la separazione dalle proprie famiglie, rimaste in Italia, sancendo talvolta un distacco definitivo, dovuto sia alla distanza fisica sia alla totale diversità di esperienze maturate.

Se di queste vicende raramente si trova traccia nella memorialistica comunista, dove gli aspetti della vita privata dei militanti vengono di norma adombrati, più ricche di rilievi personali sembrano rivelarsi le memorie e le carte private di chi, uscito in modi e tempi diversi dal Partito, dopo essersi 
dedicato per anni ad una militanza dal carattere totalizzante, avvertiva il bisogno di ripercorrere il proprio passato attraverso schemi e modelli differenti[[23]]. Era questo il caso di Angelo Tasca, noto dirigente comunista, espulso nel 1929 dal Partito, a causa del dissenso da lui maturato rispetto alla linea intrapresa dall'Internazionale.

La figura di Tasca, una delle più discusse e controverse personalità politiche dei decenni che si collocano tra il primo ed il secondo dopoguerra, è stata oggetto di diversi studi, anche grazie all'amplissimo archivio da lui lasciato, composto da carte politiche e personali metodicamente raccolte e conservate lungo il corso di una vita[[24]]. Alle diverse e successive edizioni di documenti appartenuti a Tasca[[25]], si è di recente aggiunta la pubblicazione di una serie di missive dal contenuto amoroso che, tra il 1930 e il 1931, l'allora ex dirigente comunista indirizzò da Parigi, dove viveva, ad una giovane donna di nome Cécile[[26]]. Erano quelli gli anni successivi all'espulsione dall'organizzazione comunista nei quali Tasca, che continuava a vivere nella clandestinità, si era trovato a dovere fronteggiare la perdita di tutto ciò che fino a quell'istante era stato il suo universo di riferimento[[27]]. Cécile, come rileva Anelli, era dunque divenuta per Tasca quasi un pretesto, al fine di "gettare un po' di leggerezza, sofferta, ma pur sempre tenera, soffice e appassionata, sul peso della sua storia»[[28]].

Russa, di origine ebraica, Cécile emerge da questo carteggio come costante oggetto di desideri e riflessioni ma, al tempo stesso, come figura sulla quale lo scrivente poteva proiettare i suoi sogni ripercorrendo, in una sorta di soliloquio, il proprio passato. Attraverso queste lettere, alle quali si aggiungono due missive di Cécile ed una minuta dell'amico Maurice, diviene possibile cogliere i successivi sviluppi di una tenace passione coltivata da Tasca in modo tanto intenso quanto interiorizzato.

"Ci troviamo - osserva Anelli - a spiare la vita interiore di un intellettuale che affronta una sua vicenda amorosa, privata, con la penna intinta nella passione, capace di indagini intime, insolite e rivelatrici, in uno stile letterario 
folgorante, immediato, originalissimo»[[29]]

I saggi dei due curatori forniscono poi alcuni importanti elementi di riflessione ai fini di un'analisi letteraria delle missive, pur sempre attenta al contesto di riferimento, nonché alla biografia personale e politica di chi quelle lettere aveva scritto.

Dal carteggio affiora a più riprese la tormentosa ricerca, compiuta da Tasca, di restituire alla propria esistenza e, in particolare, alla sua vita amorosa quella forte carica poetica ed estetizzante che essa pareva avere irrimediabilmente perduto. Nel rendere partecipe Cécile dei suoi pensieri e delle sue inquietudini, in un lungo corteggiamento che, a quanto pare, non era destinato a suscitare le reazioni sperate, l'ex dirigente comunista non esitava a ripercorrere i passaggi più dolorosi di una vicenda familiare irreparabilmente incrinata dalla lontananza.

"L'esilio mi ha separato dalla mia famiglia, dai miei figli, ai quali sono profondamente legato. Per lungo tempo ho sperato che la separazione fisica non mi avrebbe fatto perdere tutto. Un giorno vi parlerò di questo. Si è salvata, nel naufragio, la coscienza dei doveri verso la mia famiglia, e, anche, il bisogno di non restare solo, solo con il mio amore, che, respinto dal di fuori, si scava il suo letto nel più profondo del mio essere e s'inabissa»[[30] ].

Costretto, sin dal 1926, ad abbandonare definitivamente l'Italia, dove rimanevano la moglie Lina e i due figli, Tasca aveva seguito, al pari di altri dirigenti e funzionari comunisti, la strada di una vocazione politica intensa e totalizzante, alla quale il proprio mondo affettivo e familiare era stato completamente subordinato[[31]]. Una scelta, questa, destinata a non essere accettata dalla moglie, la quale avrebbe finito per diradare sempre più la corrispondenza diretta al marito, fino a confessare lui, dietro esplicita richiesta di spiegazioni, l'esistenza di una "nuova amicizia»[[32]]. Proprio nei mesi in cui Tasca si lasciava trasportare dall'intensa passione per Cécile, il dialogo con la moglie giungeva infatti alle battute finali, rendendo sempre più 
vane le speranze di un eventuale ricongiungimento familiare in Francia[[33]].

In simili circostanze, la figura di Cécile assumeva sempre più rilevanza e Tasca, abituato, in virtù del suo passato di "rivoluzionario", tanto alla solitudine quanto all'introspezione, doveva esprimere a più riprese il bisogno di intrattenere un dialogo che, seppur concentrato sul sentimento, rimaneva saldamente ancorato alla riflessione.

"lo non sono un "cerebrale" (lo lo sono meno di voi), i miei sentimenti non hanno bisogno di covare sotto le meningi per arrivare a schiudersi. Ho un vero odio contro ogni analisi che separa dalla vita, al posto di aprirne le porte. Ma con questo non m'abbandono all'onda di ciò che voi chiamate le "emozioni». II mio pensiero non ha mai lavorato tanto quanto dopo avervi conosciuto. Né una parola né uno sguardo va perso. Malgrado la mia emozione, assedio pazientemente la vostra vita interiore, per meglio conoscerla, per meglio avvicinarmene»[[34]]

I temi affrontati di volta in volta all'interno delle lettere consentono di cogliere la straordinaria vivacità del contesto culturale che animava Parigi all'inizio degli anni Trenta[[35]]. Tasca, come evidenzia Soave, espulso dal Pcd'I, in quel periodo aveva preso a gravitare nell'ambito del comunismo non ufficiale, prestando la propria collaborazione ad alcune riviste, tra cui "Monde", diretta da Barbusse. Crocevia di famosi letterati e di intellettuali noti per il loro costante impegno politico, quell'ambiente si dimostrava nondimeno sensibile alle sollecitazioni della psicoanalisi, che aveva gettato una luce del tutto nuova sull'universo delle pulsioni.

Nelle stesse missive dirette a Cécile, nelle quali Tasca non esitava a soffermarsi sulla natura ed i significati della sessualità, respinta, a quanto pare, dalla giovane donne a causa di uno choc infantile, è possibile avvertire il lontano eco di quel dibattito [[36]].

"Non avendo trovato quella che chiamate "la rivelazione della vita sessuale" al di fuori di voi, al momento in cui si è realizzata, la sua soddisfazione in un 
amore che vi iniziasse alla vita, siete rifluita verso il vostro passato. Si è operata allora una saldatura tra questo passato e il bisogno e la speranza dell'amore, di cui scorgevate ora il volto nuovo. La rivelazione, al posto d'allargare l'orizzonte del vostro destino, lo restringeva, dato che vi rifugiavate (per difendervi e cercarvi un alimento) in questo mondo che stava bene a voi, solo a voi, tutto per voi. Ma continuavate, così, a vivere malgrado tutto di voi stessa»[[37]]

Nonostante il rapporto di grande confidenza instauratosi tra i due, la relazione tra Tasca e Cécile pare rimanere, lettera dopo lettera, costantemente contrassegnata dall'incertezza. Cécile, infatti, benché affascinata dalla cultura nonché dalla profondità di colui che le indirizzava lettere così intense e partecipate, non doveva apparentemente ricambiare il sentimento amoroso del suo interlocutore, il quale, in talune occasioni, doveva auspicare l'interruzione dei loro rapporti, al fine di evitare ulteriori sofferenze. Mentre l'eventualità di un ricongiungimento con la propria famiglia si profilava sempre più remota, Tasca, nell'agosto del 1931, terminava, a quanto pare, la relazione epistolare con Cécile con queste parole:

"Prenderò le mie vacanze all'inizio di settembre. Se rientrate prima, potremmo dunque vederci. Ma non sarebbe meglio osservare le "distanze" ed evitarci una doppia tortura?

Non cambierete nulla ai miei sentimenti, cara Cécile, dal momento che io non vedo alcuna ragione di cambiarli» [[38]]

Ci si trova anche in tal caso d'innanzi alle complesse vicende di chi, oggetto della repressione fascista, aveva compiuto scelte di carattere politico ed esistenziale tali da modificare profondamente la propria vita privata. Grazie ai carteggi diviene quindi possibile assistere ai cambiamenti che investivano di volta in volta la sfera personale degli antifascisti, e misurare nondimeno i sacrifici nonché le sofferenze che, a causa di particolari contingenze, 
potevano accompagnare la loro militanza. Un aspetto, questo, di grande rilevanza al fine di condurre un'indagine a trecentosessanta gradi sui reali costi della scelta antifascista, superando la divisione, tanto netta quanto fittizia, tra pubblico e privato che, oltre a pervadere buona parte della memorialistica, ha qualificato parte della storiografia.

\section{Note}

[1] Vedasi, a questo proposito, i saggi contenuti in «Problemi del Socialismo», 7, 1986, numero monografico dedicato a Fascismo e antifascismo negli anni della Repubblica, e in particolare, il saggio di N. Gallerano, Critica e crisi del paradigma antifascista, 106-133. Per una esaustiva panoramica delle nuove questioni storiografiche postesi in relazione all'antifascismo si rinvia poi all'introduzione di A. De Bernardi, L'antifascismo: una questione storica aperta, a: Alberto De Bernardi, Paolo Ferrari (eds.), Antifascismo e identità europea, Roma, Carocci, 2004.

[2] Assai incisive risultano, a tal proposito, alcune osservazioni svolte nel 1978 da G. Guazza, nel saggio Storia della storiografia, storia del potere, storia sociale, in: N. Tranfaglia (ed.), L'Italia unita nella storiografia del secondo dopoguerra, Atti del convegno organizzato dalla Fondazione Feltrinelli, tenutosi a Palermo nel novembre 1978, Milano, Feltrinelli, 1980, 276-278. A questo riguardo vedasi inoltre la Prefazione di Claudio Pavone all'edizione del 1994 di Una guerra civile. Saggio storico sulla moralità nella Resistenza, Torino, Bollati Boringhieri, 1994 (prima ed. 1991). Cfr. P.G. Zunino, La Repubblica e il suo passato. II fascismo dopo il fascismo, il comunismo, la democrazia: le origini dell'Italia contemporanea, Bologna, il Mulino, 2003, 15-17.

[3] Si vedano P. Corsini, G. Porta, Avversi al regime. Una famiglia comunista negli anni del fascismo, Roma, Editori Riuniti, 1992; G. De Luna, Donne in oggetto. L'antifascismo nella società italiana. 1922-1939, Torino, Bollati Boringhieri, 1995 e P. Gabrielli, Fenicotteri in volo. Donne comuniste nel ventennio fascista 
, Roma, Carocci, 1999. Su questo filone di studi si rinvia, inoltre, a Eade, Donne nell'antifascismo, «Italia Contemporanea», 202, (marzo 1996), 99112.

[4] Si segnalano, in particolare, M. Calloni, L. Cedroni (eds.), Politica e affetti familiari. Lettere di Amelia, Carlo e Nello Rosselli a Guglielmo, Leo e Nina Ferrero e Gina Lombroso Ferrero (1917-1943), Premessa di G. Sapelli, Trascrizione e traduzione di Paola Ranzini, Milano, Feltrinelli, 1997; V. Foa, Lettere della giovinezza. Dal carcere 1935-1943, a cura di F. Montevecchi, Torino, Einaudi, 1998; Gramsci a Roma, Togliatti a Mosca. Il carteggio del 1926, a cura di C. Daniele, con un saggio di G. Vacca, Torino, Einaudi, 1999; Piero Gobetti. Carteggio 1918-1922, a cura di E. Alessandrone Perona, Torino, Einaudi, 2003.

[5] Vedasi P. Gabrielli, Mondi di carta. Lettere, Autobiografie, Memorie, Siena, Protagon Editori Toscani, 2000, 58-59. Per un'analisi delle specificità degli epistolari femminili lungo il corso dell'Età moderna si rinvia ai saggi contenuti in: G. Zarri (ed.), Per lettera. La scrittura epistolare femminile tra archivio e tipografia, secoli XV-XVII, Roma, Viella, 1999. Vedasi inoltre M. L. Betri, D. Maldini Chiarito (eds.), Dolce dono graditissimo. La lettera privata dal settecento al Novecento, Milano, Angeli, 2000.

[6] Goldoni definisce la lettera un documento del presente, nel quale però confluiscono più tempi: «il passato che vi si racconta, il presente in cui si scrive e in cui si pensa il destinatario, il futuro della ricezione, il futuro anteriore che tiene conto dei tempi intercorsi tra la scrittura e la lettura». A. Goldoni, Frammenti di autobiografia/autobiografia di frammenti, in: R. Caputo, M. Monaco (eds.), Scrivere la propria vita. L'autobiografia come problema critico e teorico, Introduzione di R. Mordenti, Atti del seminario su Scrivere di sé, tenutosi presso la Facoltà di Lettere e Filosofia di Roma II, Tor Vergata, anno accademico 1993-1994, Roma, Bulzoni, 1997, 163.

[7] Si rimanda, a questo proposito alle considerazioni di D. Maldini Chiarito, Norma e trasgressione nei carteggi dell'800, in: A. Pasi, P. Sorcinelli (eds.), Amori e trasgressioni 
. Rapporti di coppia tra ‘800 e ‘900, Bari, Dedalo, 1995, 35-37.

[8] Sul Fondo Betti-Giaccaglia vedasi anche Patrizia Gabrielli, Mondi di carta ., cit.

[9] L'inventario del fondo Betti Giaccaglia, redatto da Simona Urso, è anch'esso edito negli Annali Istituto Gramsci Emilia - Romagna, cit., 171180.

[10] Lettera di Lea Giaccaglia a Paolo Betti, Bologna, S. Giovanni in Monte, 6 agosto 1928, edita Ivi, 199.

[11] Si vedano, a questo proposito, le missive ricevute da un detenuto comunista, dalle quali Paolo Betti doveva apprendere la notizia della morte di Luce. Ivi, 192.

[12] Le lettere pubblicate, la cui scelta è stata coordinata da S. Urso, con la collaborazione di P. Gabrielli e C. Tonini, comprendono non solo parte della corrispondenza tra Paolo Betti e Lea Giaccaglia, ma anche alcune lettere di Paolo ai figli, una lettera di Luce al padre, un componimento di Vero Betti, ed alcune missive di Lea alla madre nonché le sopraccitate missive.

[13] Sull'esperienza carceraria delle detenute comuniste si rinvia a L. Mariani, Quelle dell'idea. Storie di detenute politiche. 1927-1948, Bari, De Donato, 1982.

[14] A proposito del progetto educativo perseguito, pur con diverse sfumature, da molti antifascisti risultano estremamente significative le memorie di F. Magnani, Una famiglia italiana, Milano, Feltrinelli, 1991.

[15] Lettera di Paolo Betti al figlio Vero, Civitavecchia, 7 luglio 1932, edita in «Annali Istituto Gramsci Emilia - Romagna», cit., pp. 267-268.

[16] A questo proposito si veda M. Ridolfi, Il Psi e la nascita del partito di massa. 1892-1922, Roma-Bari, Laterza, 1992.

[17] Sull'esperienza carceraria di Foa, vedasi V. Foa, Il Cavallo e la Torre. Riflessioni su una vita 
, Torino, Einaudi, 1991, 84-120

[18] Lettera di Massimo Mila alla madre Clelia Carena, 26 febbraio 1937, edita in Massimo Mila, Argomenti strettamente familiari, cit., 299-301.

[19] Lettera di Massimo Mila alla madre Clelia Carena, datata 14 giugno 1935, edita Ivi, 25-27.

[20] Lettera di Massimo Mila alla madre Clelia Carena, datata 7 febbraio 1936, edita Ivi, 172-174.

[21] Prefazione di G. Pajetta a Lettere di antifascisti dal carcere e dal confino , vol. I, Roma, Editori Riuniti, 1962, XVI. Simili le considerazioni di Amendola, il quale, a tal riguardo, nelle proprie memorie scriveva: «Le lettere dal carcere non sono mai sincere. [...]. Ho confrontato le mie lettere, che mia sorella ha conservato, con le lettere di altri carcerati politici. Prevale in tutti una rappresentazione falsa, perché eccessivamente positiva, delle condizioni di vita e di soggiorno, per non aggravare le preoccupazioni dei familiari». G. Amendola, Un'isola, Milano, Rizzoli, 1980, 102.

[22] Vedasi P. Gabrielli, Col freddo nel cuore. Uomini e donne nell'emigrazione antifascista, Roma, Donzelli, 2004.

[23] Basti qui citare I. Silone, Uscita di sicurezza, in: Ead. Uscita di sicurezza , Introduzione di B. Falcetto, Postfazione e note di M. Franzinelli, Milano, Mondadori, 2001 (ed. originale, 1949); F. Ferrero, Un nocciolo di verità, a cura di R. Farina, Milano, La Pietra, 1978; A. Spinelli, Come ho tentato di diventare saggio, vol. I, lo, Ulisse, Bologna, il Mulino 1984; B. Pizzardo, Senza pensarci due volte, Bologna, il Mulino, 1996. Cfr. P. Gabrielli, Biografie femminili e storia politica delle donne, cit. 
[24] Si veda, a questo riguardo, la nota polemica di Amendola circa l'attività di raccolta di documenti di Partito assai riservati, svolta in modo del tutto arbitrario da Tasca. G. Amendola, Un archivista nella rivoluzione, in: Ead. Comunismo, antifascismo e Resistenza, Roma, Editori Riuniti 1967, 105-132.

[25] Tra le edizioni di documenti dell'Archivio Tasca si possono qui citare: Istituto Giangiacomo Feltrinelli, La rinascita del socialismo italiano e la lotta contro il fascismo. 1934-1939. Documenti inediti dell'Archivio Angelo Tasca, Introduzione e documenti a cura di S. Merli, Milano, Feltrinelli, 1963; G. Berti, I primi dieci anni di vita del P.C.I. Documenti inediti dell'Archivio Angelo Tasca, Milano, Feltrinelli, 1967; Vichy 1940-1944: quaderni e documenti inediti di Angelo Tasca. Archives de guerre d'Angelo Tasca, a cura di D. Peschanski, Milano, Feltrinelli 1986.

[26] A. Tasca, A Cécile, a cura di S. Anelli e S. Soave, Torino, Aragno, 2001.

[27] Ricca di elementi assai importanti circa quel periodo si rivela la corrispondenza intrattenuta da Tasca con l'amico Silone, edita in D. Bidussa, Dialogato per un rinnovamento socialista. Un carteggio degli anni Trenta tra Ignazio Silone e Angelo Tasca, L'Irpinia nella società meridionale, «Annali della Fondazione Feltrinelli», (1985-1986), vol. I, 594-671.

[28] S. Anelli, Amore e psiche nelle lettre di un eretico, in A. Tasca, A Cécile, cit., 18.

[29] Ibidem.

[30] Lettera di Angelo Tasca a Cécile Beitzman, venerdì, 30 maggio [1930], edita Ivi, 22-23.

[31] Si rinvia, a questo proposito, alle riflessioni contenute nel saggio di $R$. Bodei, Il rosso, il nero, il grigio: il colore delle moderne passioni politiche, in: S. Vegetti Finzi (ed.), Storia delle passioni, Roma-Bari, Laterza, 1995, 321331.

[32] Si veda, in particolare, la lettera di Angelo Tasca a Cécile Beitzman, 11 
luglio 1930, edita in A. Tasca, A Cécile, cit., 50-54, nella quale Tasca riportava anche un brano tratto da una missiva della moglie.

[33] Si veda, a questo proposito, S. Soave, Gli anni di Cécile, Ivi, 115-119.

[34] Lettera di Angelo Tasca a Cécile Beitzman, lunedì, 30 giugno [1930], edita Ivi, 36-37.

[35] Si rinvia, a tal proposito, a H. R. Lottman, La Rive Gauche, Intellettuali e impegno politico in Francia dal Fronte popolare alla guerra fredda, Milano, Edizioni di Comunità, 1983 (ed. originale, 1981), 79-199.

[36] Si veda lo stralcio di una lettera di Maurice diretta a Cécile, 22 luglio 1930, edita in A. Tasca, A Cécile, cit., 71-72.

[37] Lettera di Angelo Tasca a Cécile Beitzman, lunedì, 7 luglio 1930, edita Ivi, 41-45. Nella parte finale della lettera, Tasca definiva «'amore di due esseri [...] un'opera d'arte, dove l'impulso sessuale entra come materia di fusione, o, se volete come un certo grado di temperatura, che assicura la fusione, ma rimane un'opera d'arte, il risultato di un'attività creatrice».

[38] Lettera di Angelo Tasca a Cécile Beitzman, 23 agosto 1931, edita Ivi, 84-85. 